\title{
Incidencia y frecuencia de la participación en línea de estudiantes universitarios mexicanos. El caso de la Universidad Veracruzana
}

\author{
Carlos A. Torres-Gastelú (1), Omar Cuevas-Salazar(2), Joel Angulo-Armenta ${ }^{(3)}$ y Agustín Lagunes-Domínguez ${ }^{(4)}$ \\ (1) Facultad de Administración, Universidad Veracruzana, Veracruz, México. (e-mail: ctorres@uv.mx) \\ (2) Departamento de matemáticas, Instituto Tecnológico de Sonora, Ciudad Obregón, Sonora, México. \\ (e-mail: ocuevas@itson.edu.mx) \\ (3) Departamento de educación, Instituto Tecnológico de Sonora, Ciudad Obregón, Sonora, México. \\ (e-mail: joel.angulo@itson.edu.mx) \\ (4) Facultad de Contaduría y Administración, Universidad Veracruzana, Veracruz, México. (e-mail: aglagunes@uv.mx)
}

* Autor a quien debe ser dirigida la correspondencia

Recibido Feb. 27, 2019; Aceptado Abr. 17, 2019; Versión final Jun. 20, 2019, Publicado Feb. 2020

\begin{abstract}
Resumen
El objetivo de este estudio fue determinar los niveles de incidencia y frecuencia de ciertas expresiones de participación en línea inherentes al ámbito social y educativo que realizan estudiantes de la Universidad Veracruzana en México. La investigación se realizó desde un enfoque cuantitativo mediante la aplicación a 628 alumnos de un instrumento con 24 indicadores evaluados a través de dos escalas de Likert, analizados mediante la estadística descriptiva e inferencial. En la muestra participaron jóvenes inscritos en alguno de los cinco programas educativos seleccionados de las dos entidades académicas elegidas, la Facultad de Administración y la Facultad de Contabilidad de la región de Veracruz. Los resultados arrojaron un bajo nivel de incidencia y frecuencia de participación en línea que realizan los jóvenes universitarios. Además, se detectó diferencias significativas entre ambos programas educativos. Sin embargo, no se detectaron diferencias significativas en las distintas formas de participación de los estudiantes por género y semestre.
\end{abstract}

Palabras clave: participación en línea; tecnologías de comunicación; ciudadanía digital; redes sociales

\section{Online participation: incidence and frequency in mexican university students. The case of the veracruzana university}

\begin{abstract}
The objective of this study was to determine the levels of incidence and frequency of certain expressions of online participation inherent in the social and educational field made by students of the Universidad Veracruzana in Mexico. The research was conducted from a quantitative approach through the application to 628 students of an instrument with 24 indicators assessed through a two Likert scale, analyzed by descriptive and inferential statistics. In the sample participated young people enrolled in one of the five educational programs selected of the two academic entities chosen: Administration and Accounting of the Veracruz region. The results show a low level of incidence and frequency of online participation by university students. In addition, no significant differences were detected in the perceptions of the students by gender and semester, although there were differences by educational program.
\end{abstract}

Keywords: online participation; communication technology; digital citizenship; social networks 


\section{INTRODUCCIÓN}

La perspectiva analítica que contempla este estudio se sustenta a partir de determinados rasgos deseables a desarrollar en los jóvenes universitarios en aras de apoyar a su proceso formativo como ciudadanos digitales. De ahí que en primer término se plantea el concepto de ciudadanía digital para posteriormente hacer una rápida revisión sobre la aproximación teórica de la participación con miras a centrarnos en el tipo de participación social en un ambiente virtual que se pretende analizar. Una vez acotado el alcance a la participación social, se presenta el constructo que alberga el mecanismo para desarrollarlo a través de las instituciones educativas: la competencia digital. Particularmente, la competencia ciudadana digital y una dimensión clave abordada en este estudio, la participación en línea. De acuerdo a Choi (2016), el concepto de ciudadanía digital se asocia con las habilidades, el pensamiento y la acción, que permiten a las personas comprender, navegar, involucrarse y transformarse a uno mismo, a la comunidad, a la sociedad y al mundo a través de la Internet. Mientras que para Arif (2016) involucra las interacciones humanas básicas y los roles emergentes de las comunidades en línea, además consiste en la capacidad para intervenir en asuntos de índole público, discutir asuntos sociales e influenciar a nivel discusivo y decisivo en una sociedad que interactúa por medio de la red.

El desarrollo de la competencia de ciudadanía digital en los jóvenes se vincula no solo al ámbito local, sino también al global (Yüksel y Eres, 2018). En la literatura se ha abordado este tema aludiendo a las características que debe tener un ciudadano global a ser desarrolladas como parte de los perfiles de egreso de los estudiantes. En este sentido, Arvanitakis y Hornsby (2016) mencionan que es recomendable que los egresados de las universidades se involucren en los procesos democráticos, en asuntos de equidad y derechos humanos, así como en la sostenibilidad ecológica para apoyar a que la sociedad funcione adecuadamente. Estos autores destacan que la nueva imperativa en la universidad se orienta en formar a los estudiantes para que sean responsables, receptivos y abiertos a realizar contribuciones informadas en línea tanto en su círculo cercano como a nivel global a través de una activa participación en línea. En resumen, lo que es sugerente formar es "un estudiante que le importa no solo obtener información y generar conocimiento sino también que está arraigado en la realidad de su contexto, orientado a los problemas e interesado en aplicar su conocimiento para el mejoramiento de la sociedad" (Arvanitakis y Hornsby, 2016: 20).

Por su parte, Robles (2011) destaca que el ciudadano digital es un tipo de ciudadano con actividades y capacidades inherentes a su entorno tanto en el desempeño que tiene en el mundo real como en el virtual. En este contexto, este estudio pretende ahondar sobre un tipo de actividad y capacidad en particular: la participación en línea. Se parte de la premisa de que el elemento de participación no se limita a un campo social específico, sino que está presente en toda sociedad y en todos los niveles (Carpentier, 2012). Considerando este esquema nos interesa abordar el campo social educativo a nivel universitario. La participación de los individuos es inherente a una expresión del poder (Carpentier, 2012), a partir de ello se propone un abordaje inicial desde la participación política para su contextualización. La participación política ha sido estudiada en al menos tres tipos: participación convencional, no convencional y social. De acuerdo a Mannarini et al. (2008) en la participación convencional el ciudadano hace uso de los canales tradicionales e institucionales para influir en las decisiones políticas. Mientras que en la participación no convencional se enfoca en atender problemas específicos como son peticiones, manifestaciones y protestas. Finalmente, la participación social tiene tres características principales: prevalencia de las relaciones horizontales e igualitarias, presencia de motivaciones pro-sociales, y una estructura de redes con conexiones débiles.

En virtud de que el objeto de estudio son las percepciones de los jóvenes universitarios que se encuentran en proceso de formación, no se consideraron ni a la participación convencional, ni a la participación no convencional. Por tanto, se parte del eje analítico de la participación social considerando algunas de las formas de expresión de actividades sociales y civiles, delimitadas a los canales de comunicación a través de la Internet como son las redes sociales virtuales y los sitios web. Es innegable que las formas de participación se han diversificado a raíz del despunte de las Tecnologías de la Información y Comunicación (TIC) encontrando una amplia gama para que los individuos se manifiesten. A medida que se han asimilado estas herramientas tecnológicas en el quehacer diario, se ha gestado una cultura digital caracterizada por el uso intensivo de herramientas y espacios en los que surgen nuevas formas para participar, pero ahora bajo la modalidad en línea. Sin embargo, la amplia difusión que tiene la Internet involucra riesgos. En este sentido, Sierra (2013) establece que el uso de las TIC, más que favorecer la presencia y participación de los ciudadanos en los asuntos colectivos, puede acabar reforzando el control y la autoridad de élites institucionales, mediante la manipulación de información, con poco margen para que se genere cambio. En otras palabras, el uso acrítico y desmesurado de las TIC podrían impulsar una virtualización encaminada a la manipulación de los usuarios, en lugar de ser un vehículo detonador del cambio social mediante la participación efectiva de los ciudadanos.

De cualquier manera, la Internet se ha constituido como el vehículo ideal para llevar a cabo este acto social virtual, colectivo, y producto de una decisión personal. Este acto social representa la voluntad personal de 
influir en la sociedad, así como la influencia de la sociedad sobre el individuo. De tal suerte que, a través de las participaciones en línea, los jóvenes negocian su identidad, aprenden habilidades sociales y se convierten en sujetos expertos en temas de su interés (Bennett, 2008). En este orden de ideas, el rol que desempeña la universidad es clave para el desarrollo de un conjunto de competencias que les serán útiles a lo largo de toda su vida. Una de estas competencias clave que todo ciudadano debe poseer, es la competencia digital, entendida como "el conjunto de conocimientos, habilidades, actitudes, estrategias y valores que se requieren cuando se utilizan las TIC y los medios digitales para realizar tareas, resolver problemas, comunicarse, gestionar información, colaborar, crear y compartir contenidos, y construir conocimiento de manera eficaz, eficiente, apropiada, crítica, creativa, autónoma, flexible, ética y reflexiva para el trabajo, el ocio, la participación, el aprendizaje, la socialización, el consumo y el empoderamiento" (Ferrari, 2012: 56). En el Marco Europeo de la Competencia Digital realizado por el Proyecto DigComp encabezado por Ferrari (2012) se establece que la competencia digital se integra por cinco áreas, que en su conjunto derivan en 21 competencias. Para los fines de este documento, atañe uno de los elementos de la competencia número 2 de comunicación: la participación ciudadana en línea.

De acuerdo al grupo de trabajo sobre la competencia digital de la Red de Bibliotecas Universitarias Españolas (REBIUN, 2016), en el marco de competencia digital para estudiantes universitarios se propone que la competencia de participación social mediante tecnologías digitales se refiere a: (1) participar en la sociedad mediante el uso de servicios digitales públicos y privados; y (2) buscar oportunidades para el autoempoderamiento y la participación activa en la universidad y en la sociedad en general, mediante las tecnologías digitales apropiadas. Los niveles de competencia de participación social en los jóvenes universitarios ocurren de dos formas: inicial y medio. En el nivel inicial se espera que el joven universitario conozca espacios y servicios de participación en red (dentro de la universidad y de la sociedad en general), en los que interviene de forma esporádica. En tanto, en el nivel medio, además de cumplir con lo establecido en el nivel inicial, el joven universitario localiza y selecciona servicios de participación en red en función de su utilidad. Participa activamente en comunidades virtuales y redes sociales con fines de actualización y desarrollo académico y profesional.

La competencia digital de participación social es desarrollada a través de un conjunto de conocimientos, habilidades y actitudes. Con relación a los conocimientos, es recomendable que los jóvenes: sepan que la tecnología se puede utilizar para la participación en acciones académicas y administrativas dentro de la Universidad; sepan que la tecnología se puede utilizar para la participación en acciones democráticas; y conozcan cómo las tecnologías y los medios de comunicación pueden facilitar diferentes formas de participación social. Mientras que las habilidades sugeridas tienen que ver con que sean capaces de encontrar y acceder a una serie de comunidades, redes y medios sociales dedicados a la participación social. Así como saber y poder utilizar las distintas funcionalidades de las comunidades, redes y medios sociales dedicados a la interacción social. Finalmente, en cuanto a las actitudes, es sugerente que los jóvenes estén conscientes del potencial que tienen las tecnologías y los medios digitales para la participación social. Además, es sugerente que los jóvenes tengan una comprensión crítica de los medios sociales de comunicación y difusión digitales, redes y comunidades en línea (REBIUN, 2016).

Ahora bien, en el año 2016, la Sociedad Internacional para la Tecnología en la Educación (ISTE), por sus siglas en inglés, se dio a la tarea de revisar los estándares para el uso de la tecnología en la educación generando la tercera versión de los Estándares ISTE para estudiantes con el propósito de preparar a los jóvenes para el futuro. Estos estándares enfatizan las habilidades y competencias que se desean deberían ser desarrollados en los estudiantes para permitirles participar y prosperar en un mundo digital conectado; uno de los siete componentes de estos estándares y que atañe a este estudio es el de Ciudadano Digital. La Competencia de Ciudadanía Digital de acuerdo a estos estándares se suscita cuando "los estudiantes reconocen los derechos, responsabilidades y oportunidades de vivir, aprender y trabajar en un mundo digital interconectado, y actúan de manera segura, legal y ética" (ISTE, 2016: 1). De acuerdo a la norma es deseable que los estudiantes: Participen en un comportamiento positivo, seguro, legal y ético cuando se utiliza la tecnología, incluyendo las interacciones sociales en línea o cuando se utilizan dispositivos en red.

A partir de los lineamientos propuestos por el marco Europeo de la Competencia de Ciudadanía Digital, así como de las habilidades y competencias deseables a desarrollar en los estudiantes de cualquier nivel elaborados por ISTE, se propone el constructo de manifestación de la ciudadanía digital aplicable a los jóvenes universitarios desarrollado en el marco de un proyecto de investigación que buscó en una primera etapa identificar la percepción de este colectivo. En el entendido de que el constructo de la manifestación de la ciudadanía digital no cubre a todos sus componentes detectados en la literatura, sino sólo aquellos que pueden ser susceptibles a ser desarrollados en el contexto universitario. La manifestación Ciudadana Digital, comprende los modos de expresión sobre las participaciones en línea publicadas en distintos ámbitos, las dinámicas de seguridad y privacidad digital, el apego a normas de comportamiento ético en las dinámicas de interacciones virtuales, así como los hábitos desarrollados hacia la gestión de datos personales que realizan 
los individuos cuando están conectados a la red. El alcance de este documento contempla a la primera dimensión de estudio: Participación en línea. Esta dimensión atiende a las formas de intervención en línea que realizan los estudiantes universitarios en comunidades digitales, redes sociales, sitios web con el fin de publicar sugerencias, propuestas, demandas de corte social, económico, político en sus círculos sociales privados o públicos. De ahí que el acotamiento del constructo de participación en línea se refiere a las acciones que realizan dichos jóvenes en su contexto universitario.

A lo largo del texto se alude al término de participación ciudadana circunscrito exclusivamente a las interacciones que los jóvenes universitarios realizan cuando están conectados a la Internet, a pesar de que el concepto es más amplio y comprende a las acciones que se realizan en el mundo real. Ahora bien, el estudio se limitó a identificar solamente las formas de expresión en línea en tres dimensiones específicas: Presencia en comunidades digitales universitarias, apoyo a propuestas virtuales de cambio, y publicación de opiniones en la red. Sobre estas tres dimensiones, se pretende señalar el nivel de incidencia y frecuencia de participación en línea que realizan los jóvenes universitarios en el sur de México, así como las diferencias en la percepción de los encuestados considerando a las variables de programa educativo, sexo y semestre; para finalmente identificar el tipo de correlación que exista entre la incidencia y frecuencia de las diversas formas de participación en línea.

\section{OTROS ANTECEDENTES}

En México existen diversos estudios que han abordado las prácticas que realizan los jóvenes universitarios en la Internet (Padilla y Flores, 2011; Padilla, 2014; Meneses et al., 2013; Meneses y Castillo, 2016; Meneses et al., 2017; Reguillo, 2003; Ribeiro et al., 2017). En el estudio de Padilla y Flores (2011), identificaron y analizaron las prácticas políticas que realizan los jóvenes universitarios utilizando como soporte mediático la Internet, con relación a las instancias políticas formales. Estos autores destacan que existe un alejamiento de los jóvenes en su participación en las prácticas políticas formales, aunque sí manifiestan interés en los problemas públicos, así como en el desempeño del poder y el gobierno. En un estudio posterior de Padilla (2014) sobre la misma temática, detectó que las prácticas políticas que realizan los jóvenes universitarios en Aguascalientes se quedaron en el nivel más superficial, en donde se identificaron posturas y reflexiones, pero no se dio una participación política que se tradujera en acciones y resultados concretos. A pesar de ello, la manera como la Internet empodera a los jóvenes en relación con su ciudadanía política según Padilla (2014), es que representa una importante herramienta para conformar comunidades en la red, aunque no con fines de participación política. Por lo que el acceso y uso de la Internet por parte de los jóvenes no se asocia con una mayor participación ciudadana, ni tampoco implica un cambio favorable para una participación ciudadana democrática. Sugiere que se requiere emprender una mejor formación de los jóvenes respecto de su uso de la Internet, es decir, una alfabetización mediática y una formación ciudadana.

En el estudio realizado por Meneses et al. (2013) cuyo objetivo fue conocer los hábitos y perspectivas de los estudiantes universitarios de la Ciudad de México en torno a la participación política, la libertad de expresión y el uso de redes sociales. Sus hallazgos señalan que las redes sociales fungen sólo como espacios de circulación informacional y exposición a flujos de datos, y no tanto como herramientas para participar. Al parecer la juventud permanece al margen de actividades relevantes para la vida pública y para su propio desarrollo humano. Aunado a ello, se encuentra la brecha digital en México y la incipiente cultura cívica que prevalece. Aunque se han vislumbrado algunos esfuerzos en el desarrollo de las redes de indignación, tal como ocurrió en la historia reciente de México con el movimiento estudiantil \#YoSoy132 (Meneses, 2015). El movimiento \#YoSoy132 emergió frente a una situación nacional marcada por una ola de violencia que se extiende por todo el territorio y ante una coyuntura electoral manipulada por los medios. Este movimiento que surgió en el nivel universitario se configura como una opinión pública supranacional y representó una expresión de participación de los estudiantes. En un estudio posterior de Meneses y Castillo (2016), se evidencia la labor de participación sobre el caso de desapariciones forzadas en Ayotzinapa México entre septiembre y diciembre de 2014. Nuevamente a través de las redes sociales digitales, y particularmente en el hashtag \#Todossomos Ayotzinapa se manifestó la indignación en la sociedad debido a estos acontecimientos. Los movimientos sociales estudiados por estos autores destacan el poder de las redes sociales en un contexto de escasa confianza institucional, estrecha pluralidad mediática e incipiente cultura participativa.

En otro estudio realizado por Meneses et al. (2017) destacan que las redes sociales propician una ruta alterna confiable para que los jóvenes se expresen, dialoguen y se movilicen. Además, reiteran que no todos los jóvenes mexicanos están conectados en línea, y que no todos los jóvenes participan en las redes virtuales de una manera significativa para el desarrollo humano. De ahí que el rol de la universidad es clave en el proceso de inclusión digital y desarrollo de las habilidades, conocimientos y actitudes de los jóvenes hacia las diferentes formas de participación en línea que pueden realizar en el día a día en su quehacer como ciudadanos digitales. En este sentido, Ribeiro et al. (2017) manifiestan que existen signos de apatía política y desconfianza de ciudadanos en general, con un especial énfasis en la juventud. 
En la revisión de la literatura internacional se detectaron diversos estudios que abordan las diversas formas de participación digital y el desarrollo de la competencia de ciudadanía digital (Haav, 2018; Choi, 2016; Clark, 2009; Khurana et al., 2015; Odetti y Caldeiro, 2016; Zúñiga y Duque, 2009; Montaña et al., 2012; Gladwell, 2010; Avalos, 2014; Casacuberta y Gutiérrez-Rubí, 2010; Mossberger et al., 2007; Campos y Silván, 2012; Piscitelli et al., 2010; Yuste, 2015; Livingstone et al., 2012; Gavilan et al., 2017; Cano-Correa et al., 2017; Granados-Romero et al., 2014).

De estos estudios detectados realizados en otros países, algunos han abordado la construcción de la ciudadanía digital (Odetti y Caldeiro, 2016; Haav, 2018; Casacuberta y Gutiérrez-Rubí, 2010; Choi, 2016; Clark, 2009; Khurana et al., 2015). El trabajo realizado por Odetti y Caldeiro (2016) está orientado a aproximarse al significado atribuido por estudiantes al tema de la participación y la ciudadanía. En tanto, Haav (2018) resalta que las universidades requieren redoblar esfuerzos para propiciar una participación activa de los jóvenes en diversos ámbitos (cívica, política, social, económica, legal y cultural) para que en todo momento protejan sus derechos, privacidad y seguridad digital. De la misma manera, Casacuberta y Gutiérrez-Rubí (2010) destacan que los canales digitales se han conformado como un recurso válido y útil para la participación en línea que realizan los ciudadanos digitales ante organismos y las autoridades mediante el uso de múltiples herramientas tecnológicas propiciando no solo la recepción de la información, sino también la emisión de información. Por lo tanto, ahora más que nunca los jóvenes necesitan apoyo para desarrollar una ciudadanía socialmente responsable en la era de la Internet (Choi, 2016; Clark, 2009; Khurana et al., 2015).

En la revisión de la literatura se detectaron diversas formas de participación en línea que realizan los jóvenes (Avalos, 2014; Mossberger et al., 2007; Campos y Silván, 2012). Avalos (2014) aborda otras formas de participación en línea en los jóvenes como son las protestas por medios digitales como un mecanismo para manifestar las opiniones, inconformidades, reportar eventos sociales violentos o bien injusticias sociales cuestionando la autonomía y legitimidad del sistema político ante las situaciones que publican. Por su parte, Mossberger et al. (2007) proponen que la ciudadanía digital puede ser medida por las actividades políticas y económicas que los individuos realizan en línea. De tal manera que los ciudadanos digitales necesitan desarrollar la habilidad para participar en la sociedad en línea y requiere un acceso regular y efectivo a la Internet, así como las habilidades para usar esta tecnología, esto es, la competencia digital. En el estudio realizado por Campos y Silván (2012), se centraron en la participación activa de los ciudadanos en los asuntos públicos, en sus círculos sociales inmediatos y en el rol que desempeñan en espacios virtuales abiertos partiendo de las acciones y hábitos que realizan cotidianamente en las interacciones que realizan con sus pares, con la sociedad y con las autoridades.

Con relación a las formas de participación en sitios web, autores como Zúñiga y Duque (2009) estudiaron la interactividad en medios digitales colombianos y coincidieron en caracterizar un bajo aprovechamiento de sus propiedades en relación con los usuarios. Otro tipo de participación virtual con posturas poco deseables y negativas fuera de los alcances de este estudio tiene que ver con el ciberodio en los foros de noticias caracterizados por el insulto y ultraje (Montaña et al., 2012), alejándose de los esquemas de participación ética responsable que debe regir a todo ciudadano digital. De la otra cara de la moneda, se encuentran los estudios (Gladwell, 2010) que señalan los beneficios del uso de las tecnologías como impulsores de la participación en ámbitos virtuales propiciando diversas escalas dependiendo de la herramienta tecnológica, como el rol del Twitter por ser más apto para la movilización en comparación con el blog que promueve más a fondo los espacios deliberativos de manera efectiva (Gladwell, 2010), o bien el Facebook que resulta adecuado para la difusión y el crecimiento de los debates ciudadanos.

Por su parte, la literatura sobre los estudios relacionados con la participación de los ciudadanos en la Internet resalta la subutilización que están teniendo estos canales de comunicación emergentes (Piscitelli et al., 2010; Yuste, 2015; Livingstone et al., 2012; Gavilan et al., 2017; Cano-Correa et al., 2017). De la misma manera, otros autores (Granados-Romero et al., 2014) señalan que a pesar de que la tecnología ha permeado en la sociedad actual existe un rezago en términos de las formas y posibles potencialidades sobre su uso, particularmente en lo referente a las formas como éstas pueden ser usadas para participar en línea. En este sentido, Piscitelli et al. (2010) sostienen que las formas de participación de los ciudadanos en las redes sociales están caracterizadas por una amplia disparidad representada por aquellos que deciden indicar que una noticia o publicación les gusta, otros que optan por reenviarla, unos tantos que se decantan por comentarla o incluso otros que contribuyen aportando nuevos materiales. Ante esta subutilización de los canales digitales, Yuste (2015), destaca la necesidad del desarrollo de competencias en los jóvenes universitarios que permitan el adecuado uso de las herramientas tecnológicas y la conformación de pensamiento crítico para para expresarse, compartir, informarse, debatir, y organizarse en la red, y particularmente en las redes sociales. Autores como Livingstone et al. (2012), establecen una tendencia en los jóvenes adultos a ejercer su rol como mero observador pasivo ante la información a la que tienen acceso, denotando poca actividad que realizan en redes sociales como indicio de la falta de aprovechamiento de la 
totalidad de sus potencialidades. En este mismo orden de ideas, Gavilan et al. (2017:68), encontraron "una reducida capacidad para influir en el entorno, crear agenda y movilizar a la sociedad". Estos autores proponen una tipología de jóvenes representada por pro-digitales, moderadores duales y escépticos totales, siendo los primeros los que reflejaron mayor actividad, aunque fueron la minoría.

\section{HIPÓTESIS DE TRABAJO}

Las hipótesis planteadas en este estudio fueron las siguientes:

$\mathrm{H}_{1}$ : Las formas de participación en línea que realizan estudiantes universitarios presentan un bajo nivel de incidencia.

$\mathrm{H}_{2}$ : Existe diferencia significativa en la incidencia de las formas de participación en línea que realizan estudiantes universitarios entre los cinco programas educativos seleccionados de la Universidad Veracruzana.

$\mathrm{H}_{3}$ : Existe diferencia en la incidencia de las formas de participación en línea que realizan las mujeres y los hombres adscritos al área económico-administrativa en la región Veracruz de la Universidad Veracruzana.

$\mathrm{H}_{4}$ : No hay relación en la incidencia de las formas de participación en línea que realizan estudiantes universitarios entre los semestres escolares que comprenden a los programas educativos seleccionados de la Universidad Veracruzana.

$\mathrm{H}_{5}$ : Las formas de participación en línea que realizan estudiantes universitarios presentan un bajo nivel de frecuencia.

$\mathrm{H}_{6}$ : Existe diferencia significativa en la frecuencia de las formas de participación en línea que realizan estudiantes universitarios entre los cinco programas educativos que se seleccionaron en la región Veracruz de la Universidad Veracruzana.

$\mathrm{H}_{7}$ : Existe diferencia significativa en la frecuencia de las formas de participación en línea que realizan las mujeres y los hombres adscritos a las dos facultades de la región Veracruz de la Universidad Veracruzana.

$\mathrm{H}_{8}$ : No hay relación en la frecuencia de participación en línea que realizan estudiantes universitarios entre los semestres escolares que comprenden a los programas educativos seleccionados de la Universidad Veracruzana.

$\mathrm{H}_{9}$ : Existe una correlación positiva entre las percepciones de incidencia y frecuencia sobre las formas de participación en línea que manifestaron los jóvenes universitarios de la Universidad Veracruzana.

\section{MATERIALES Y MÉTODOS}

El objetivo del presente estudio fue determinar los niveles de incidencia y frecuencia de ciertas expresiones de participación en línea que realizan estudiantes universitarios de la Universidad Veracruzana en México. Para ello, se utilizó un enfoque de corte cuantitativo, con un diseño no experimental transeccional. El tipo de muestreo fue no probabilístico bajo el criterio por conveniencia. En el estudio participaron 628 alumnos de los siguientes programas educativos: Administración (28.3\%), Administración Turística (15.9\%), Contabilidad (37.1\%), Gestión de Negocios (9.1\%) y Sistemas Computacionales Administrativos (8.9\%), en la región Veracruz de la Universidad Veracruzana en México. El $59.1 \%$ son mujeres y $40.9 \%$ hombres, en tanto la edad promedio fue de 20.5 años. El mayor porcentaje de alumnos se concentró en el sexto semestre con $21.7 \%$, mientras que el menor porcentaje de alumnos fue de $0.2 \%$ del noveno semestre. Se administraron dos instrumentos relacionados con la participación en línea por parte de los alumnos. El primero para identificar la incidencia de la participación en línea, con 13 preguntas (Tabla 1) y opciones de respuesta tipo Likert, (1) No me describe, (2) Me describe poco, (3) Me describe y (4) me describe mucho.

Tabla 1: Listado de ítems de incidencia (Inci) y de frecuencia (Frec) del instrumento de participación en línea.

\begin{tabular}{|c|c|c|c|}
\hline Dimensiones & Inci. & Frec. & Descripción del ítem \\
\hline \multirow{4}{*}{$\begin{array}{l}\text { Dim } 1 \\
\text { Presencia en } \\
\text { comunidades } \\
\text { digitales } \\
\text { universitarias }\end{array}$} & 1 & & $\begin{array}{l}\text { Pertenezco a alguna comunidad digital en la que publico mis sugerencias, } \\
\text { propuestas o demandas relativas a mi universidad }\end{array}$ \\
\hline & 2 & & $\begin{array}{l}\text { Considero que la universidad me ha ayudado a conocer mis derechos y } \\
\text { obligaciones como ciudadano digital }\end{array}$ \\
\hline & 3 & 1 & $\begin{array}{l}\text { Participo en actividades o consultas lanzadas por la Universidad mediante sus } \\
\text { redes sociales o sitio web }\end{array}$ \\
\hline & 4 & 2 & $\begin{array}{l}\text { Participo en actividades, consultas o propuestas generadas por el propio alumnado } \\
\text { mediante grupos no oficiales de la universidad }\end{array}$ \\
\hline \multirow{4}{*}{$\begin{array}{l}\text { Dim } 2 \\
\text { Apoyo a } \\
\text { propuestas } \\
\text { virtuales de } \\
\text { cambio }\end{array}$} & 5 & 3 & $\begin{array}{l}\text { Elaboro propuestas de cambio social, económico o político en plataformas } \\
\text { especializadas (Por ejemplo: Change.org) }\end{array}$ \\
\hline & 6 & 4 & $\begin{array}{l}\text { Participo en propuestas de cambio social, económico o político lanzados desde } \\
\text { plataformas especializadas (Por ejemplo: Change.org) }\end{array}$ \\
\hline & 7 & 5 & Apoyo con mi firma a iniciativas solidarias a través de la Internet \\
\hline & 8 & 6 & Formo parte de programas de voluntariado surgidos en la red \\
\hline
\end{tabular}


Tabla 1 (continuación)

\begin{tabular}{|l|c|c|l|}
\hline \multirow{2}{*}{\begin{tabular}{l} 
Dim 3 \\
\multirow{2}{*}{$\begin{array}{l}\text { Publicación de } \\
\text { opiniones en la } \\
\text { red }\end{array}$}
\end{tabular}} & 9 & 7 & Manifiesto en línea mi opinión sobre demandas sociales \\
\cline { 2 - 4 } & 11 & 8 & Participo en debates sobre asuntos públicos en sitio web \\
\cline { 2 - 4 } & 12 & 10 & $\begin{array}{l}\text { Expreso mis opiniones en línea con mi círculo cercano de contactos (Facebook, } \\
\text { Twitter, Instagram, etc.) }\end{array}$ \\
\cline { 2 - 5 } & 13 & 11 & Expreso mis opiniones en línea con desconocidos (sitio de discusión, blogs, etc.) \\
\hline
\end{tabular}

Se realizó análisis factorial utilizando el método de factorización de ejes principales con rotación ortogonal Oblimin con Kaiser mostrando un total de tres factores que explican el $56.2 \% \%$ de la varianza total de los puntajes. El primer factor es el más importante y explica el $36.2 \%$ de la varianza; el segundo, explica 11.5\%, y el tercer factor explica el 8.5\%. El primer factor está constituido por 4 variables (ítems 5, 6, 7 y 8 ), las cuales se pueden identificar como "Apoyo a propuestas virtuales de cambio"; el segundo factor consta de 4 variables (ítems 1, 2, 3 y 4) y refleja la "Presencia en comunidades digitales universitarias"; y, el tercero, agrupa 5 variables (ítems 9, 10,11, 12 y 13) las cuales están relacionadas con "Publicación de opiniones en la red". Sólo por fines de orden y en lo sucesivo, la dimensión 1 estará compuesta por las preguntas de la 1 a la 4; la dimensión 2 por las preguntas de la 5 a la 8 ; y la dimensión 3 por las preguntas de la 9 a la 13. Para determinar la pertinencia del análisis factorial se obtuvo la medida de adecuación muestral de Kaiser-Meyer-Olkin (KMO) que fue de .870 lo que indica que se justifica el uso del análisis factorial con los datos muestrales.

Además, se realizó la prueba de esfericidad de Bartlett, obteniendo un valor $p$ prácticamente de cero, lo que indica la existencia de correlación entre las variables analizadas. La confiabilidad del instrumento con los 13 ítems se obtuvo utilizando el Alfa de Cronbach, el cual fue de .840 lo que refleja una consistencia interna elevada. El segundo instrumento se utilizó para identificar la frecuencia de participación en línea en los estudiantes, el cual consistió de 11 ítems (tabla 1) con opciones de respuesta tipo Likert, (1) Nunca, (2) Muy pocas veces, (3) Algunas veces, (4) Casi siempre y (5) Siempre. Se realizó el análisis factorial, obteniéndose prácticamente los mismos resultados en cuanto a los ítems por dimensiones. La varianza explicada por los tres factores fue $67.8 \%$. La confiabilidad obtenida de este instrumento fue de 0.872 .

El procedimiento que se siguió para el desarrollo del presente estudio fue el siguiente: (1) Se contactó a los directores de las facultades de Administración y Contabilidad para explicarles el propósito del estudio; (2) Se solicitó la base de datos de los grupos de alumnos de las Facultades y se seleccionaron los grupos que se les aplicaría el instrumento. Solo fueron incluidos aquellos alumnos que por voluntad propia decidieron participar y dieron su consentimiento; (3) Se aplicó el instrumento en formato impreso y se capturó la información para su análisis; (4) Se utilizó estadística descriptiva e inferencial, específicamente distribución de frecuencias y gráficos, así como correlación y pruebas de hipótesis. Se utilizaron pruebas estadísticas no paramétricas como la U de Mann-Whitney y de Kruscal Wallis; para ello se usó el programa estadístico informático para las ciencias sociales (SPSS).

\section{RESULTADOS Y DISCUSIÓN}

En primer término, se muestran los resultados del instrumento relacionado con la incidencia de participación en línea. Los ítems que presentaron mejor incidencia tomando en cuenta las opciones de respuesta "Me describe mucho" y "Me describe" son; la pregunta 12 "Expreso mis opiniones en línea con mi círculo cercano de contactos" con $50.5 \%$, la pregunta 2 "Considero que la universidad me ha ayudado a conocer mis derechos y obligaciones como ciudadano digital" con $43.2 \%$, la pregunta 3 "Participo en actividades o consultas lanzadas por la Universidad mediante sus redes sociales o sitio web" con $39.2 \%$ y la pregunta 4 "Participo en actividades, consultas o propuestas generadas por el propio alumnado mediante grupos no oficiales de la universidad" con $35.2 \%$. La dimensión 1 "Presencia en comunidades digitales universitarias" presenta mayor incidencia de participación por parte de los alumnos con $35.4 \%$ para las opciones de respuesta "Me describe mucho" y "Me describe" (Figura 1).

El menor porcentaje de respuestas de "Me describe mucho" y "Me describe" es para la pregunta 8 "Formo parte de programas de voluntariado surgidos en la red" con $12.4 \%$, seguido de las preguntas 5 "Elaboro propuestas de cambio social, económico o político en plataformas especializadas" y la 10 "Participo en debates sobre asuntos públicos en sitio web" con el mismo porcentaje $12.6 \%$ y después la pregunta 13 "Expreso mis opiniones en línea con desconocidos" con 13.3\%. La dimensión 2 "Apoyo a propuestas virtuales de cambio" es la que presenta el nivel más bajo de incidencia en la participación de los alumnos con un $17.1 \%$ (Figura 1). La incidencia global de todo el instrumento se muestra también en la figura 1 y fue de $25 \%$, la cual representa un porcentaje bajo. 


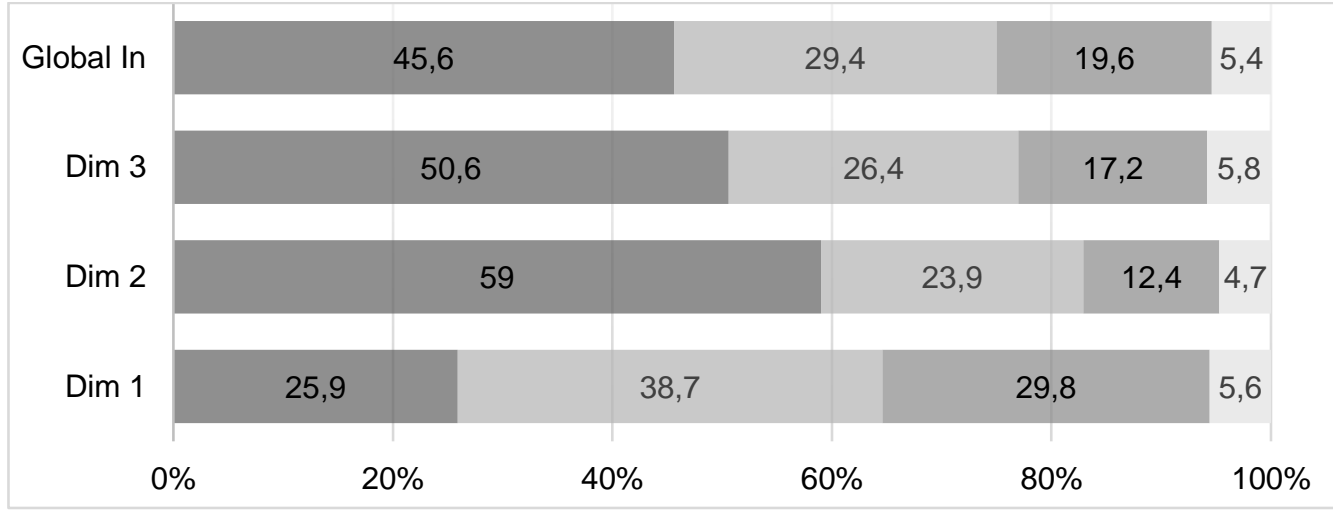

Fig. 1: Distribución de la incidencia de la participación en línea de los alumnos: Global (Global In) y por dimensión

Otra forma de analizar la escala tipo Likert y con la intención de establecer niveles de incidencia, es obteniendo puntuaciones promedio de acuerdo al número de participantes que contestaron la encuesta. Puesto que la puntuación más grande en la escala es de 4 y son 628 participantes, se tendrá una puntuación máxima de 2512. Este valor, se dividirá entre la suma obtenida (por pregunta) de las respuestas de todos los participantes. Para efectos de este estudio, se considera el siguiente criterio: si la puntuación promedio es de 1 a 1.5 se tendrá un nivel de incidencia muy baja por parte de los alumnos; de 1.6 a 2.0, la incidencia será baja; de 2.1 a 3.0 la incidencia será moderada; de 3.1 a 3.5 la incidencia será alta y, de 3.6 a 4 muy alta.

Los resultados se distribuyen de acuerdo a la tabla 2: Cuatro preguntas con incidencia muy baja, cinco preguntas con incidencia baja y cuatro preguntas con incidencia moderada y ninguna con incidencia alta 0 muy alta. Considerando el instrumento de manera global, la incidencia fue de 1.8, que, de acuerdo al criterio, es una incidencia baja. De esto se deduce que las formas de participación en línea que realizan estudiantes universitarios presentan un bajo nivel de incidencia. Es decir, se acepta la hipótesis $\mathrm{H}_{1}$, las formas de participación en línea que realizan estudiantes universitarios presentan un bajo nivel de incidencia. Estos hallazgos coinciden con lo señalado por Granados-Romero et al. (2014) en términos del rezago sobre las formas y posibles potencialidades de uso de la tecnología en la sociedad.

Tabla 2: Agrupación de ítems por nivel de incidencia.

\begin{tabular}{|c|c|c|c|c|c|}
\hline Criterio & 1.0 a 1.5 & 1.6 a 2.0 & 2.1 a 3.0 & 3.1 a 3.5 & 3.6 a 4.0 \\
\hline İtems & $5,8,10$ y 13 & $1,6,7,9,11$ & $2,3,4,12$ & Ninguno & Ninguno \\
\hline
\end{tabular}

Con relación a la hipótesis $\mathrm{H}_{2}$ sobre si existe diferencia significativa en la incidencia de las formas de participación en línea que realizan estudiantes universitarios entre los cinco diferentes programas educativos participantes del estudio, se utilizó la prueba Kruskal Wallis, con un nivel de significancia de 0.05 . Los resultados indicaron que hubo diferencia significativa sólo en 4 de las 13 preguntas, en las preguntas 1, 2, 12 y 13. Los alumnos del programa de Sistemas de Computación Administrativos tienen mayor incidencia en cinco $(1,9,10,11$ y 13), que en su mayoría pertenecen a la dimensión 3 de "Publicación de opiniones en la red"; los alumnos de Contaduría muestran mayor incidencia en la pregunta 2; los alumnos de Administración Turística tienen mayor incidencia en las preguntas 2 y 12, los alumnos del programa de educativo de Gestión de Negocios resultaron con mayor incidencia en cinco $(3,5,6,7$ y 8$)$ preguntas, que en su mayoría pertenecen a la dimensión 2 "Apoyo a propuestas virtuales de cambio" y por último, la facultad de Administración en ninguna resultó con mayor incidencia. Los resultados de la prueba estadística fueron significativos reflejando diferencias entre los estudiantes de los cinco programas educativos. Por tanto, se acepta la hipótesis $\mathrm{H}_{2}$ de que existe diferencia significativa en la incidencia de las formas de participación en línea que realizan estudiantes universitarios entre los cinco programas educativos que se seleccionaron en la región Veracruz de la Universidad Veracruzana. Los alumnos de los programas de Sistemas Computacionales Administrativos y de Gestión de Negocios muestran mejor incidencia en la participación en línea.

Para probar la hipótesis $\mathrm{H}_{3}$ referente a si la incidencia de las formas de participación en línea es la misma por género, es decir, entre hombres y mujeres, se utilizó la prueba $U$ de Mann-Whitney, con un nivel de significancia de 0.05 . Los resultados indicaron que no hubo diferencia significativa en ninguna de las preguntas. La prueba resultó no significativa, es decir, no hay diferencias entre las percepciones de hombres y mujeres. Por lo tanto, se rechaza la hipótesis $\mathrm{H}_{3}$ de que existe diferencia significativa en la incidencia de las formas de participación en línea que realizan las mujeres y los hombres adscritos al área económicoadministrativa en la región Veracruz de la Universidad Veracruzana. 
En cuanto a la hipótesis $\mathrm{H}_{4}$, se analizó si había presencia de correlación entre el semestre en el que estaba inscrito el alumno y sus formas de participación en línea, para ello se utilizó la prueba de Spearman, con un nivel de significancia de 0.05 . Los resultados indicaron presencia de correlación negativa en la pregunta 2 "Considero que la universidad me ha ayudado a conocer mis derechos y obligaciones como ciudadano digital". Esto es, los alumnos de semestres más avanzados consideran que la universidad le ha ayudado en menor medida a conocer sus derechos y obligaciones en la participación digital. No hubo presencia de correlación entre la incidencia de las formas de participación en línea (considerando todo el instrumento) y el semestre que cursa el alumno. De ahí que se acepte la hipótesis $\mathrm{H}_{4}$, no hay relación en la incidencia de las formas de participación en línea que realizan estudiantes universitarios entre los semestres escolares que comprenden a los programas educativos seleccionados de la Universidad Veracruzana.

Se analizó el segundo instrumento de 11 ítems para identificar la frecuencia de la participación en línea por parte de los alumnos, para ello se obtuvo la distribución de frecuencias de cada uno de los ítems, así como del instrumento de manera global. Tomando en cuenta las opciones de respuesta de "casi siempre" y "siempre", la menor frecuencia de participación resultó en el ítem 6 "Formo parte de programas de voluntariado surgidos en la red" con un porcentaje de 3.7, seguido de la pregunta 3 "Elaboro propuestas de cambio social, económico o político en plataformas especializadas" con un 3.8\% y después la pregunta 8 "Participo en debates sobre asuntos públicos en sitio web" con un 5.4\%. La que presentó mayor porcentaje de frecuencia de participación fue el ítem 10 "Expreso mis opiniones en línea con mi círculo cercano de contactos" con un $31.2 \%$ de las mismas dos opciones de respuesta. La frecuencia de participación del instrumento global resultó en un $10.7 \%$.

Los niveles de la frecuencia de participación en línea se obtuvieron con las puntuaciones promedio de acuerdo al número de participantes que contestaron la encuesta. Puesto que la puntuación más grande en la escala es 5 y son 628 participantes, se tendrá una puntuación máxima de 3140 . Este valor, se dividirá entre la suma obtenida (por pregunta) de las respuestas de todos los participantes. Se considera el siguiente criterio: si la puntuación promedio es de 1 a 1.5 se tendrá un nivel de frecuencia muy bajo por parte de los alumnos; de 1.6 a 2.5, la incidencia será baja; de 2.6 a 3.5 la incidencia será moderada; de 3.6 a 4.5 la incidencia será alta $y$, de 4.6 a 5 muy alta.

En la tabla 3 se muestra la distribución por criterio: Cuatro preguntas con frecuencia baja y siete preguntas con frecuencia moderada y ninguna con frecuencia alta o muy alta. Considerando el instrumento de manera global, el nivel de frecuencia fue de 2.8, que, de acuerdo al criterio, es una frecuencia baja. De esto se deduce que las formas de participación en línea que realizan estudiantes universitarios presentan un bajo nivel de frecuencia. Es decir, se acepta la hipótesis $\mathrm{H}_{5}$, Las formas de participación en línea que realizan estudiantes universitarios presentan un bajo nivel de frecuencia. Los hallazgos de este estudio reiteran la subutilización de los recursos tecnológicos que ofrece la Internet (Piscitelli et al., 2010; Yuste, 2015; Livingstone et al., 2012; Gavilan et al., 2017; Cano-Correa et al., 2017), manifestado de acuerdo a los resultados presentados en este documento, por el bajo nivel de frecuencia en las formas de participación en línea que realizan estudiantes universitarios.

Tabla 3: Agrupación de ítems por nivel de frecuencia.

\begin{tabular}{|c|c|c|c|c|c|}
\hline Criterio & 1.0 a 1.5 & 1.6 a 2.5 & 2.6 a 3.5 & 3.6 a 4.5 & 4.6 a 5.0 \\
\hline Ítems & Ninguno & $1,2,5,10$ & $3,4,6,7,8,9,11$ & Ninguno & Ninguno \\
\hline
\end{tabular}

Con relación a la hipótesis $\mathrm{H}_{6}$ sobre si existe diferencia significativa en la frecuencia de las formas de participación en línea que realizan estudiantes universitarios entre los cinco programas educativos participantes, se utilizó la prueba Kruskal Wallis, con un nivel de significancia de 0.05. Los resultados indicaron que hubo diferencia significativa en 5 de las 11 preguntas, la 2, 3, 5, 8 y 10. Los alumnos del programa de Gestión de Negocios tienen mayor nivel de frecuencia en seis ítems $(5,6,7,8,10$ y 11$)$ de las 11 preguntas, que en su mayoría pertenecen a la dimensión 3 de "Publicación de opiniones en la red"; los alumnos de Administración Turística tienen mayor incidencia en las preguntas 1 y 2, las cuales forman la dimensión 1 "Presencia en comunidades digitales universitarias"; y, los alumnos del programa de Sistemas Computacionales Administrativos" resultaron con mayor incidencia en tres ( 3,4 y 9 ) preguntas. La prueba general de todo el instrumento resultó significativa, es decir, hay diferencias entre los cinco programas educativos. Por tanto, se acepta la hipótesis $\mathrm{H}_{6}$, existe diferencia significativa en la frecuencia de las formas de participación en línea que realizan estudiantes universitarios entre los cinco programas educativos que se seleccionaron en la región Veracruz de la Universidad Veracruzana. Los alumnos de los programas de Sistemas Computacionales Administrativos y de Gestión de Negocios muestras mayor frecuencia en la participación en línea. 
Referente a la hipótesis $\mathrm{H}_{7}$ para probar si la frecuencia de participación en línea es la misma entre hombres y mujeres, se utilizó la prueba $U$ de Mann-Whitney, con un nivel de significancia de 0.05 . Los resultados indicaron que hubo diferencia significativa en un solo ítem, el 11 "Expreso mis opiniones en línea con desconocidos", donde las mujeres muestran mayor nivel de frecuencia al intercambiar opiniones con desconocidos. La prueba general de todo el instrumento resultó no significativa, es decir, no hay diferencias entre las percepciones de hombres y mujeres en la frecuencia de participación. Por lo tanto, se rechaza la hipótesis $\mathrm{H}_{7}$, existe diferencia significativa en la frecuencia de las formas de participación en línea que realizan las mujeres y los hombres adscritos a las dos facultades de la región Veracruz de la Universidad Veracruzana.

Para la hipótesis $\mathrm{H}_{8}$ en cuanto a si había presencia de correlación entre el semestre en el que estaba inscrito el alumno, con respecto a la frecuencia de participación en línea, se utilizó la prueba de Spearman con un nivel de significancia de 0.05 . Los resultados no indicaron presencia de correlación alguna. No hubo presencia de correlación entre la frecuencia de participación en línea y el semestre que cursa el alumno, en ninguna de las 11 preguntas, ni en el instrumento considerado de forma global. De ahí que se acepte la hipótesis $\mathrm{H}_{8}$, no hay relación en la frecuencia de participación en línea que realizan estudiantes universitarios entre los semestres escolares que comprenden a los programas educativos seleccionados de la Universidad Veracruzana.

Finalmente, para la hipótesis $\mathrm{H}_{9}$ sobre la posible concordancia entre las percepciones de incidencia y frecuencia sobre las formas de participación en línea que manifestaron los jóvenes, se realizaron pruebas de correlación para determinar la existencia de relación entre los ítems del instrumento de incidencia y los ítems del instrumento de frecuencia, es decir, se probó si el ítem 3 del instrumento de incidencia estaba relacionado con el ítem 1 del instrumento de frecuencia, el 4 con el 2 y así sucesivamente; para ello se utilizó la prueba de Spearman con un nivel de significancia de 0.05 . Los resultados indicaron la presencia de correlación en las 11 pruebas, con un valor $p$ menor que 0.001 en todas ellas. Es decir, a mayor incidencia de formas de participación en línea, mayor frecuencia en la participación en línea.

\section{CONCLUSIONES}

La participación en línea es un componente clave a ser considerado en el proceso formativo de los ciudadanos digitales responsables. El estudio de la incidencia y frecuencia de las acciones que realizan los jóvenes permiten valorar su grado de madurez en los comportamientos cuando navegan en línea. Los indicadores utilizados en este estudio se vinculan a prácticas sugeridas que promueven el desarrollo de la competencia de ciudadanía digital restringida al ámbito de la participación en línea para un contexto de jóvenes en proceso de formación profesional. A partir de los resultados obtenidos, se concluye que: 1) las formas de participación en línea que realizan estudiantes universitarios presentan un bajo nivel de incidencia y frecuencia; 2) existe diferencia significativa en la incidencia y frecuencia de las formas de participación en línea que realizan estudiantes universitarios entre los cinco programas educativos seleccionados; 3) no existe diferencia significativa en la incidencia y frecuencia de las formas de participación en línea que realizan las mujeres y los hombres; 4) no hay relación en la incidencia y frecuencia de las formas de participación en línea que realizan estudiantes universitarios entre los semestres escolares que comprenden a los programas educativos seleccionados; y 5) existe una correlación positiva entre las percepciones de los estudiantes universitarios sobre sus formas de participación en línea tanto en la incidencia como en la frecuencia, lo cual denota una marcada consistencia entre las acciones que los alumnos dicen realizar con la frecuencia que reportan.

\section{AGRADECIMIENTOS}

Los autores agradecemos las facilidades otorgadas para el desarrollo del presente trabajo al Consejo Nacional de Ciencia y Tecnología (CONACyT) en México. Así como a las autoridades, docentes y alumnos de la Universidad Veracruzana para la realización del proyecto "La perspectiva del estudiante universitario hacia la Ciudadanía Digital".

\section{REFERENCIAS}

Arif, R., Internet as a Hope or a Hoax for Emerging Democracies: Revisiting the Concept of Citizenship in the Digital Age, doi: 10.1016/j.sbspro.2016.12.002, Procedia - Social and Behavioral Sciences, 236, 4-8 (2016)

Arvanitakis, J. y D.J. Hornsby, Universities, the Citizen Scholar and the Future of Higher Education, $1^{\text {a }}$ Ed., 15-20. Palgrave MacMillan, Hampshire, UK (2016)

Avalos, J.M., Disidencias Juveniles y Medios Digitales en México: ¿Una Coyuntura con Elementos de Futuro para la Participación Política?, Argumentos, ISSN: 0187-5795, 27(75), 147-170, (2014)

Bennett, W.L., Civic Life Online: Learning How Digital Media Can Engage Youth, 2a Ed., 1-206. MIT Press, Cambridge, MA, USA (2008) 
Campos, E. y A. Silván, Democracia Digital. El Estado de la Cuestión, I. Ramos y E. Campos (coords): Ciudadanía en 3D. Democracia Digital Deliberativa. Un Análisis Exploratorio. Editorial Edhasa, Barcelona, España (2012)

Cano-Correa, A.M., M.T. Quiroz-Velasco y R. Nájar-Ortega, Jóvenes Universitarios en Lima: Política, Medios y Participación, doi: 10.3916/C53-2017-07, Comunicar, XXV(53), 71-79 (2017)

Carpentier, N., The Concept of Participation. If they have Access and Interact, do they Really Participate, doi: 10.4013/fem.2012.142.10, Revista Fronteiras, 14(2), 164-177 (2012)

Casacuberta, D. y A. Gutiérrez-Rubí, E-Participación de cómo las Nuevas Tecnologías están Transformando la Participación Ciudadana, Razón y Palabra, ISSN: 1605-4806, 15(73) (2010)

Choi, M., A Concept Analysis of Digital Citizenship for Democratic Citizenship Education in the Internet Age, doi: 10.1080/00933104.2016.1210549, Theory \& Research in Social Education, 44(4), 565-607 (2016)

Clark, L., Focus Group Research with Children and Youth, doi: 10.1111/j.1744-6155.2009.00187.x, Journal for Specialists in Pediatric Nursing, 14(2), 152-4 (2009)

Ferrari, A., Digital Competence in Practice: An Analysis of Frameworks, doi: 10.2791/82116, JRC Scientific and Policy Reports (2012)

Gavilan, D., G. Martínez-Navarro y S. Fernández-Lores, Universitarios y Redes Sociales Informativas: Escépticos Totales, Moderados Duales o Pro-Digitales, doi: 10.3916/C53-2017-06, Comunicar, XXV(53), 61-70 (2017)

Gladwell, M., Small Change: Why the Revolution Will Not Be Tweeted, The New Yorker, 4, $42-49$ (2010)

Granados-Romero, J., R. López-Fernández y otros cuatro autores, Las Tecnologías de la Información y las Comunicaciones, las del Aprendizaje y del Conocimiento y las Tecnologías para el Empoderamiento y la Participación como Instrumentos de Apoyo al Docente de la Universidad el Siglo XXI, Medisur, ISSN: 1727-897X, 12(1), 289-294, (2014)

Haav, K., European Identity and Citizenship in Estonia: Analyses of Textbooks and Theoretical Developments, doi: 10.4119/UNIBI/jsse-v17-i2-1906, Journal of Political Science Education, 17(2), 67-79 (2018)

ISTE Standards for Students (ebook). A Practical Guide for Learning with Technology. ISTE, USA (2016)

Khurana, A., A. Bleakley, A.B. Jordan y D. Romer, The Protective Effects of Parental Monitoring and Internet Restriction on Adolescents' Risk of Online Harassment, doi: 10.1007/s10964-014-0242-4, Empirical Research, 44(5), 1039-1047 (2015)

Livingstone, S., L. Haddon y A. Görzig, Children, Risk and Safety on the Internet: Research and Policy Challenges in Comparative Perspective, $1^{\text {a }}$ Ed., 1-408. Policy Press, Bristol, UK (2012)

Mannarini, T., M. Legittimo y C. Taló, Determinants of Social and Political Participation Among Youth. A Preliminary Study, Psicología Política, ISSN: 1138-0853, 36, 95-117, (2008)

Meneses, M.E., Ciberutopías: Democracia, Redes Sociales y Movimientos Red, $1^{\text {a }}$ Ed., 1-145. Editorial Porrúa México / Tecnológico de Monterrey (2015)

Meneses, M.E., E. Ortega y G.A. Urbina, Jóvenes, Participación Político Ciudadana y Redes Sociales en México 2012, La Libertad de Expresión en el Proceso Electoral de 2012. PNUD/COPARMEX/ITESM-CCM/Porrúa, pp 10-13, México (2013)

Meneses, M.E., E. Ortega y G.A. Urbina, Youth and Political Participation. \#Yosoy132 and the Struggle for Freedom of Expression in Mexico, doi: 10.15581/003.30.1.87-103, Communication \& Society, 30(1), 87-103 (2017)

Meneses, M.E. y M.C. Castillo, \#TodossomosAyotzinapa, Storytelling, Identidades, Representaciones y Reflexividad en Disputa, doi: 10.6035/clr.2016.16.3, Revista de Estudios Culturales de la Universitat Jaume I, XXVI, 37-56 (2016)

Montaña, S., V. González y C. Ariza, Ciberodio en los Comentarios de las Noticias en Línea en Colombia. El Caso de Piedad Córdoba, doi: 10.5294/3367, Palabra Clave, 16(3), 812-841 (2012)

Mossberger, K., C.J. Tolbert y R.S. McNeal, Digital Citizenship: The Internet, Society, and Participation., 234-240. Mlt Press, Massachusetts, Estados Unidos (2007)

Odetti, V. y G. Caldeiro, Cultura Juvenil y Participación en el Espacio Público Digital desde la Perspectiva de Estudiantes Secundarios, II Bienal Iberoamericana de Infancias y Juventudes, 1-9 (2016)

Padilla, M.R., Ciudadanía Política en la Red: Análisis de las Prácticas Políticas entre Jóvenes Universitarios, Comunicación y Sociedad, ISSN: 0188-252X, (21), 71-100, (2014)

Padilla, M.R. y D. Flores, El Estudio de las Prácticas Políticas de los Jóvenes en Internet, doi: 10.32870/cys.v0i15.1141, Comunicación y Sociedad, (15), 101-122 (2011)

Piscitelli, A., I. Adaime y I. Binder, El Proyecto Facebook y la Posuniversidad. Sistemas Operativos Sociales y Entornos Abiertos de Aprendizaje, 1aㅡ. Ed., 1-261. Editorial Ariel, Colección Fundación Telefónica, Madrid, España (2010)

Reguillo, R., Ciudadanías Juveniles en América Latina, doi: 10.4067/S0718-22362003000200002, Última Década, 11(19), $11-30$ (2003)

REBIUN Red de Bibliotecas Universitarias Españolas, Marco de Competencia Digital para Estudiantes de Grado: Adaptación de DIGCOMP, Estudios e Informes Línea 22016 (2016) 
Ribeiro, N., T. Neves y E. Menezes, An Organization of the Theoretical Perspectives in the Field of Civic and Political Participation: Contributions to Citizenship Education, doi: 10.1080/15512169.2017.1354765, Journal of Political Science Education, 13(4), 426-446 (2017)

Robles, J.M., Ciudadanía Digital: Una Introducción a un Nuevo Concepto de Ciudadano, 1ª Ed,1-120. UOC, Barcelona, España (2011)

Sierra, F., Ciudadanía, Tecnología y Cultura. Nodos Conceptuales para la Nueva Mediación Digital, 1르 Ed, 1-320. Comunicación. Serie Comunicología Latina. Gedisa, España (2013)

Yuste, B., Las Nuevas Formas de Consumir Información de los Jóvenes. Revista de Estudios de Juventud, ISSN: 0211 4364, 15(108), 179-191, (2015)

Yüksel, A. y F. Eres, The Correlation Between Global Citizenship Perceptions and Cultural Intelligence Levels of Teachers, doi: 10.13189/ujer.2018.060528, Universal Journal of Educational Research, 6(5), 1069-1076 (2018)

Zúñiga, D. y A.E. Duque, Las Herramientas Interactivas de Dos Diarios Colombianos, Signo y Pensamiento, ISSN: $0120-$ 4823, 28(54), 330-340, (2009) 